\title{
Cromotubación histeroscópica: técnica alternativa para la evaluación de la permeabilidad tubárica y la cavidad uterina en un solo paso
}

\author{
Fernando Gómez C. ${ }^{1}$, Claudia Camila Giraldo P. ${ }^{2}$ \\ ${ }^{1}$ Unidad de Medicina Reproductiva, Servicio de Ginecología, Clínica de Marly, Bogotá. Colombia. ${ }^{2}$ Fellow en Medicina \\ Reproductiva, Fundación Universitaria Sanitas, Bogotá. Colombia.
}

\section{RESUMEN}

La evaluación de la permeabilidad tubárica es un paso esencial en el estudio de la infertilidad femenina. Existen varios métodos que permiten realizar esta evaluación. La histerosalpingografía (HSG) se ha considerado el método estándar, sin embargo es conocida su limitación en la evaluación de la obstrucción proximal con alta tasa de falsos positivos. Presentamos una técnica alternativa para la evaluación por medio de cromotubación histeroscópica, que tiene entre otras, la ventaja de permitir la evaluacion del factor uterino y tubárico en un solo paso y además, supera la dificultad de los falsos positivos de la HSG, en cuanto a la obstrucción proximal.

\section{PALABRAS CLAVE: Histeroscopia, infertilidad, trompas uterinas, salpingografía, histerosalpingografía}

\section{SUMMARY}

The evaluation of tubal patency is an essential step in the study of female infertility. There are several methods to perform this evaluation. Hysterosalpingography (HSG) has been considered the standard method, however it is known to limitations in the assessment of proximal obstruction with high false positive rate. We present an alternative technique for evaluation by hysteroscopic chromo tubation, which has among others, the advantage of allowing the evaluation of uterine and tubal factor in one step and also overcomes the difficulty of false-positive HSG, as to the proximal obstruction.

\section{KEY WORDS: Hysteroscopy, infertility, fallopian tube, salpingography, hysterosalpingography}

\section{INTRODUCCIÓN}

Según datos publicados por el Instituto Nacional de Excelencia Clínica del Reino Unido (NICE) en 2013, la infertilidad de origen tubárico, representa aproximadamente el $20 \%$ de todos los casos de infertilidad (1). Probablemente en algunos escenarios, representa un porcentaje aún mayor. Por esta razón, la valoración de la permeabilidad tubárica, sigue siendo un punto importante en la evaluacion inicial de la pareja infértil (2).

En muchos programas, la valoración tubárica hace parte del estudio inicial de cualquier mujer con subfertilidad $(2,3)$. En otros, se realiza después de dos o tres intentos de inducción de ovulación sin lograr embarazo. En todo caso, siempre que nos enfrentamos a una historia de infertilidad, nos interrogamos a cerca de la permeabilidad y competencia 
de las trompas uterinas, como órganos esenciales para el logro de un embarazo espontáneo $(3,4)$.

La evaluacion de la fertilidad, incluida la evaluacion de la permeabilidad tubárica, pretende ubicar a las parejas en uno de tres tipos de tratamiento: médico, quirúrgico o técnicas de reproducción asistida.

Los métodos para la evaluación de la permeabilidad tubárica, muchas veces pueden ser complementarios y no mutuamente excluyentes $(5,6)$. Muy a menudo, el diagnóstico exacto y el tratamiento efectivo, requiere el uso de más de una de las técnicas disponibles:

Histerosalpingografia (HSG): Ha sido el método estándar para evaluar las trompas uterinas y algunas publicaciones han mostrado, que puede ofrecer ciertos beneficios terapéuticos $(7,8)$. Los valores predictivo positivo (VPP) y predictivo negativo (VPN) de la HSG son 38 y $94 \%$ respectivamente $(5,6,8)$. Los hallazgos que sugieren una obstrucción proximal, pueden requerir más evaluación para descartar artefactos que produzcan contracciones miometriales o tubáricas transitorias, o relacionadas con la posición del catéter. Si la HSG indica permeabilidad, es muy improbable la obstrucción. Sin embargo, hasta en $60 \%$ de las mujeres en quienes la HSG inicial, muestra obstrucción proximal, una HSG repetida un mes después, muestra permeabilidad (7).

Sonohisterografía (SHG). Básicamente es una ecografía pélvica transvaginal utilizando un medio hídrico para la evaluación. Permite evaluar la cavidad uterina y la permeabilidad tubárica por evaluacion ecográfica de la presencia de líquido en la cavidad pélvica, pero no permite distinguir entre permeabilidad uni o bilateral $(9,10)$.

Canulación tubárica por histeroscopia. Utilizando fluoroscopio, confirma o excluye cualquier obstrucción tubárica sugerida por una HSG o laparoscopia y ofrece medios para una posible corrección quirúrgica por medio de recanalización utilizando catéteres especiales (11).

Laparoscopia con cromotubación. Permite identificar las trompas permeables e identificar precisamente, el sitio de la obstrucción y al mismo tiempo validar opciones de manejo quirúrgico. Sin embargo, generalmente requiere ingreso al hospital y siempre anestesia general $(9,10,12,13)$.

El objetivo de esta comunicación es presentar 6 pacientes de una serie de casos con historia de infertilidad, en cuyo estudio se requirió la realización de histeroscopia, y exponer una técnica alternativa para la evaluación de la permeabilidad tubárica con cromotubación, durante la realización de la histeroscopia.

\section{PACIENTES Y MÉTODO}

Las seis pacientes descritas, corresponden a casos en estudio de infertilidad, que en su proceso diagnóstico requirieron la realización de histeroscopia oficinal y quienes además tenían HSG realizada en los dos años anteriores. Durante la realización de la histeroscopia, se realiza prueba de cromotubación y se aplican criterios para definir permeabilidad u obstrucción. Se realizó la comparación de los hallazgos, con lo reportado en la HSG.

El procedimiento se realiza en el escenario oficinal, con histeroscopio de Bettochi y usando como medio de distensión, solución salina 0,9\%. La histeroscopia, realizada con la técnica convencional de consultorio, "sin tocar", es decir, sin pinzamiento del cérvix y con mínimo contacto del instrumental con los tejidos. Se realiza navegación en vagina, oclusión manual del orificio vulvar, identificación del orificio cervical externo y acceso al canal endocervical, momento en el cual se puede omitir la oclusión de la vulva, ingreso a cavidad uterina y valoración de la misma en la forma convencional: fondo, caras anterior, posterior, laterales y ostium bilateralmente. Luego se introduce a través del canal del histeroscopio, una cánula de $0,3 \mathrm{~mm}$, hidrofílica y flexible, la cual se dirige hacia el ostium y se ingresa en la trompa $1 \mathrm{a} 2 \mathrm{~cm}$. Se realiza inyección de $10 \mathrm{cc}$ de azul de metileno y se observa si ocurre retorno del colorante hacia la cavidad uterina. Simultáneamente, con ecografía transabdominal, se evalúa la salida de líquido perianexial en el lado que se está evaluando. Posteriormente, se realiza igual procedimiento en la trompa contralateral.

Comparamos los resultados obtenidos, con los resultados de HSG previas, de las pacientes y en forma adicional, se midió la experiencia de dolor durante el procedimiento por medio de escala visual análoga (EVA) entre 0 y 10. A continuación se presenta la serie de 6 pacientes infértiles en estudio (Tabla I):

Caso 1. Paciente de 32 años, infertilidad secundaria de 2 años de evolución. Sospecha de mioma tipo 2 o 3, por lo cual se le indica histeroscopia. Tiene HSG del año anterior que informó obstrucción tubárica proximal derecha. Mediante la cromotubación histeroscópica se accedió al segmento proximal de ambas trompas. No hubo reflujo de colorante a la cavidad uterina y se observó líquido perianexial bilateral por ecografía. Se consideró permeabilidad tubárica bilateral. EVA 3/10.

Caso 2. Paciente de 35 años, infertilidad primaria. Antecedente de endometriosis y cirugía pélvica para resección de endometrioma derecho. HSG previa informó obstrucción tubárica distal bilateral. Se realizó histeroscopia por sospecha de pólipo endometrial en ecografía transvaginal. La cromotubación mostró reflujo de colorante hacia el útero a través de ambos ostium. No se observó salida de líquido a la cavidad pélvica. Se consideró obstrucción tubárica bilateral. EVA 5/10. 


\section{Tabla I \\ CASOS DE CROMOTUBACIÓN HISTEROSCÓPICA, COMPARACIÓN CON HISTEROSALPINGOGRAFÍA PREVIA (HSG)}

\begin{tabular}{|c|c|c|c|c|c|c|}
\hline \multirow[b]{2}{*}{ Caso } & \multirow[b]{2}{*}{ Edad } & \multicolumn{4}{|c|}{ CROMOTUBACION HISTEROSCÓPICA } & \multirow[b]{2}{*}{ HSG } \\
\hline & & Reflujo color & Hidroperitoneo & $\begin{array}{l}\text { EVA } \\
0-10\end{array}$ & Interpretación & \\
\hline 1 & 32 & Ninguno & Bilateral & $3 / 10$ & Permeabilidad bilateral & $\begin{array}{l}\text { Obstrucción proximal } \\
\text { derecha }\end{array}$ \\
\hline 2 & 35 & Bilateral & No & $5 / 10$ & Obstrucción bilateral & $\begin{array}{l}\text { Obstrucción distal } \\
\text { bilateral }\end{array}$ \\
\hline 3 & 29 & Ninguno & Bilateral & $4 / 10$ & Permeabilidad bilateral & $\begin{array}{l}\text { Permeabilidad } \\
\text { bilateral }\end{array}$ \\
\hline 4 & 33 & Trompa derecha & Anexo izquierdo & $3 / 10$ & Obstrucción derecha & $\begin{array}{l}\text { Obstrucción distal } \\
\text { derecha }\end{array}$ \\
\hline 5 & 30 & Ninguno & Bilateral & $2 / 10$ & Permeabilidad bilateral & $\begin{array}{l}\text { Obstrucción proximal } \\
\text { bilateral }\end{array}$ \\
\hline 6 & 33 & Trompa izquierda & Anexo derecho & $3 / 10$ & Obstrucción izquierda & $\begin{array}{l}\text { Obstrucción proximal } \\
\text { derecha y distal } \\
\text { izquierda }\end{array}$ \\
\hline
\end{tabular}

Caso 3. Paciente de 29 años, infertilidad secundaria. Pérdida recurrente de la gestación. HSG previa informó permeabilidad tubárica bilateral. Se realiza histeroscopia diagnóstica. La cromotubación mostró hallazgos compatibles con permeabilidad tubaria bilateral. EVA 4/10.

Caso 4. Paciente de 33 años, infertilidad primaria. Hemorragia uterina anormal tipo hipermenorrea. HSG previa informó obstrucción tubárica distal derecha, trompa izquierda permeable. La cromotubación histeroscópica, muestra reflujo de colorante hacia el útero a través del ostium derecho, no hay líquido en topografía anexial derecha. Trompa izquierda sugiere permeabilidad. EVA 3/10.

Caso 5. Paciente de 30 años, con infertilidad primaria. HSG previa sugiere obstrucción tubárica bilateral proximal. Se realiza histeroscopia que muestra cavidad uterina normal, moco sobre ostium izquierdo. Se realiza cromotubación, no hay reflujo de colorante hacia la cavidad y se observa paso de líquido hacia la topografía anexial bilateralmente. EVA 2/10.

Caso 6. Paciente de 33 años. Infertilidad primaria. Historia de endometriosis, liberación de adherencias por laparoscopia. HSG del año previo informó obstrucción tubárica bilateral, hidrosalpinx izquierdo y obstrucción proximal derecha. La histeroscopia mostró pólipo móvil sobre ostium derecho, la cromotubación revela reflujo de colorante a través del ostium izquierdo. No hay reflujo por ostium derecho, hidroperitoneo en la topografía anexial izquierda.

\section{DISCUSIÓN}

La evaluación de la permeabilidad tubárica, es un paso esencial en la evaluación inicial de la infertilidad femenina. Existen varios métodos válidos para la valoración tubárica, sin embargo es fundamental hacer una selección adecuada del método más indicado en cada caso, según las intervenciones y estudios que requiera cada paciente. Cuando el escenario, corresponde a un caso en el que se requiera histeroscopia, o en los grupos en los que la histeroscopia se realiza en forma sistemática para la evaluación y estudio de la infertilidad femenina, la cromotubación realizada en el mismo acto, ofrece una alternativa adecuada y bien tolerada para tal valoración.

\section{CONCLUSIÓN}

La cromotubación histeroscópica, se propone como un método alternativo para la valoración del factor tubárico en el estudio de la infertilidad. En un mismo procedimiento se valora la cavidad uterina y la permeabilidad tubárica. Es un procedimiento bien tolerado en el escenario oficinal o de consultorio, y permite franquear los falsos positivos de la HSG en cuanto a la obstrucción proximal tubárica. Esta se constituye en una forma práctica y fiable para evaluar el factor uterino y tubárico en un mismo momento, dentro del algoritmo de estudio de la infertilidad femenina. 


\section{REFERENCIAS}

1. National Institute for Health and Clinical Excellence (NICE). Assessment and treatment for people with fertility problems, 2013. Nice clinical guideline 156. Disponible en: www.nice.org.uk.

2. The Practice Committee of the American Society for Reproductive Medicine. Optimal evaluation of the infertile female. Fertil Steril 2012;86:S264-7.

3. Homburg R, Insler V (2002) Ovulation induction in perspective. Hum Reprod Update 2002;8:449-62.

4. Thessaloniki ESHRE/ASRM-Sponsored PCOS Consensus Workshop Group. Consensus on infertility treatment related to polycystic ovary syndrome. Fertil Steril 2008;89:505-22.

5. Honore GM, Holden AE, Schenken RS. Pathophysiology and management of proximal tubal blockage. Fertil Steril 1999;5:785-95.

6. Evers JL, Land JA, Mol BW. Evidence-based medicine for diagnostic questions. Semin Reprod Med 2003;21:9-15.

7. Dessole S, Meloni GB, Capobianco G, Manzoni MA, Ambrosini G, Canalis GC. A second hysterosalpingography reduces the use of selective technique for treatment of a proximal tubal obstruction. Fertil Steril 2000;73:1037-9.

8. Johnson $\mathrm{N}$, Vanderkerchove $\mathrm{P}$, Lilford $\mathrm{R}$, Harada $\mathrm{T}$, Hughes $\mathrm{E}$, Luttjeboer $\mathrm{F}$, et al. Tubal flushing for subfertility. Cochrane Database Syst Rev 2009;1:CD003718.

9. Tulandi T, Marzal A. Redefining reproductive surgery. J Minim Invasive Gynecol 2012;19:296-306.

10. Hamed HO, Shahin AY, Elsamman AM. Hysterosalpingo-constrast sonography versus radiographic hysterosalpingography in the evaluation of tubal patency. Int J GynecolObstet 2009;105:215-7.

11. Papaioannou S, Afnan M, Coomarasamy A, Ola B, Hammadieh N, Temperton DH, et al. Long term safety of fluoroscopically guide selective salpingography and tubal catheterization. Hum Reprod 2002;17:370-2.

12. Farhi J, Ben-Haroush A, Lande Y, Fisch B. Role of treatment with ovarian stimulation and intrauterine insemination in women with unilateral tubal occlusion diagnosed by hysterosalpingography. Fertil Steril 2007;88:396-400.

13. Practice Committee of the American Society for Reproductive Medicine. Salpingectomy for hydrosalpinx prior to in vitro fertilization. Fertil Steril 2008;90:S66-8. 